\title{
Research Article \\ Quasicone Metric Spaces and Generalizations of Caristi Kirk's Theorem
}

\author{
Thabet Abdeljawad $^{1}$ and Erdal Karapinar ${ }^{2}$ \\ ${ }^{1}$ Department of Mathematics, Çankaya University, 06530 Ankara, Turkey \\ ${ }^{2}$ Department of Mathematics, Atılım University, 06836 Ankara, Turkey \\ Correspondence should be addressed to Thabet Abdeljawad, thabet@cankaya.edu.tr
}

Received 4 July 2009; Accepted 3 December 2009

Recommended by Hichem Ben-El-Mechaiekh

Cone-valued lower semicontinuous maps are used to generalize Cristi-Kirik's fixed point theorem to Cone metric spaces. The cone under consideration is assumed to be strongly minihedral and normal. First we prove such a type of fixed point theorem in compact cone metric spaces and then generalize to complete cone metric spaces. Some more general results are also obtained in quasicone metric spaces.

Copyright (c) 2009 T. Abdeljawad and E. Karapinar. This is an open access article distributed under the Creative Commons Attribution License, which permits unrestricted use, distribution, and reproduction in any medium, provided the original work is properly cited.

\section{Introduction and Preliminaries}

In 2007, Huang and Zhang [1] introduced the notion of cone metric spaces (CMSs) by replacing real numbers with an ordering Banach space. The authors there gave an example of a function which is contraction in the category of cone metric spaces but not contraction if considered over metric spaces and hence, by proving a fixed point theorem in cone metric spaces, ensured that this map must have a unique fixed point. After that series of articles about cone metric spaces started to appear. Some of those articles dealt with the extension of certain fixed point theorems to cone metric spaces (see, e.g., [2-5]), and some other with the structure of the spaces themselves (see, e.g., $[3,6]$ ). Very recently, some authors have used regular cones to extend some fixed point theorems. For example, in [7] a result about Meir-Keeler type contraction mappings has been extended to regular cone metric spaces. In other works, some results about fixed points of multifunctions on cone metric spaces with normal cones have been obtained as well [8]. For the use of lower semicontinuous functions in obtaining fixed point theorems in cone metric spaces we refer to [9].

In this manuscript, we use cone-valued lower semicontinuous functions to extend some of the results in Caristi [10] and Ekeland [11] to CMS and quasicone metric space (QCMS). The cones under consideration are assumed to be strongly minihedral and normal 
and hence regular. In particular the cone $P=[0, \infty)$ in the real line $\mathbb{R}$ is strongly minihedral and normal; hence the results mentioned in the above references are recovered.

Throughout this paper $E$ stands for a real Banach space. Let $P:=P_{E}$ always be a closed subset of E. $P$ is called cone if the following conditions are satisfied:

(C1) $P \neq \emptyset$,

(C2) $a x+b y \in P$ for all $x, y \in P$ and non-negative real numbers $a, b$,

(C3) $P \cap(-P)=\{0\}$ and $P \neq\{0\}$.

For a given cone $P$, one can define a partial ordering (denoted by $\leq$ : or $\leq_{P}$ ) with respect to $P$ by $x \leq y$ if and only if $y-x \in P$. The notation $x<y$ indicates that $x \leq y$ and $x \neq y$ while $x \ll y$ will show $y-x \in \operatorname{int} P$, where int $P$ denotes the interior of $P$. From now on, it is assumed that $\operatorname{int}(P) \neq \emptyset$.

The cone $P$ is called

(N) normal if there is a number $K \geq 1$ such that for all $x, y \in E$,

$$
0 \leq x \leq y \Longrightarrow\|x\| \leq K\|y\|
$$

$(R)$ regular if every increasing sequence which is bounded from above is convergent. That is, if $\left\{x_{n}\right\}_{n \geq 1}$ is a sequence such that $x_{1} \leq x_{2} \leq \cdots \leq y$ for some $y \in E$, then there is $x \in E$ such that $\lim _{n \rightarrow \infty}\left\|x_{n}-x\right\|=0$.

In $(N)$, the least positive integer $K$, satisfying (1.1), is called the normal constant of $P$. Note that, in $[1,2]$, normal constant $K$ is stated a positive real number, $(K>0)$. However, later on and in [2, Lemma 2.1] it was proved that there is no normal cone with constant $K<1$.

Lemma 1.1. (i) Every regular cone is normal.

(ii) For each $k>1$, there is a normal cone with normal constant $K>k$.

(iii) The cone $P$ is regular if every decreasing sequence which is bounded from below is convergent.

The proof of (i) and (ii) were given in [2] and the last one just follows from definition.

Example 1.2 (see [2]). Let $E=C^{1}[0,1]$ with the norm $\|f\|=\|f\|_{\infty}+\left\|f^{\prime}\right\|_{\infty}$, and consider the cone $P=\{f \in E: f \geq 0\}$.

For each $k \geq 1$, put $f(x)=x$ and $g(x)=x^{2 k}$. Then, $0 \leq g \leq f,\|f\|=2$ and $\|g\|=2 k+1$. Since $k\|f\|<\|g\|, k$ is not normal constant of $P$ and hence $P$ is a nonnormal cone.

Definition 1.3. Let $X$ be a nonempty set. Suppose that the mapping $d: X \times X \rightarrow E$ satisfies the following:

(M1) $0 \leq d(x, y)$ for all $x, y \in X$,

(M2) $d(x, y)=0$ if and only if $x=y$,

(M3) $d(x, y) \leq d(x, z)+d(z, y)$, for all $x, y \in X$. 
Then $d$ is said to be a quasicone metric on $X$, and the pair $(X, d)$ is called a quasicone metric space (QCMS). Additionally, if $d$ also satisfies

$$
(M 4) d(x, y)=d(y, x) \text { for all } x, y \in X,
$$

then $d$ is called a cone metric on $X$, and the pair $(X, d)$ is called a cone metric space (CMS).

Example 1.4. Let $E=\mathbb{R}^{3}$ and $P=\{(x, y, z) \in E: x, y, z \geq 0\}$ and $X=\mathbb{R}$. Define $d: X \times X \rightarrow E$ by $d(x, \tilde{x})=(\alpha|x-\tilde{x}|, \beta|x-\tilde{x}|, \gamma|x-\tilde{x}|)$, where $\alpha, \beta, \gamma$ are positive constants. Then $(X, d)$ is a CMS. Note that the cone $P$ is normal with the normal constant $K=1$.

Definition 1.5. Let $(X, d)$ be a CMS, $x \in X$, and let $\left\{x_{n}\right\}_{n \geq 1}$ be a sequence in $X$. Then

(i) $\left\{x_{n}\right\}_{n \geq 1}$ converges to $x$ if for every $c \in E$ with $0 \ll c$ there is a natural number $N$, such that $d\left(x_{n}, x\right) \ll c$ for all $n \geq N$. It is denoted by $\lim _{n \rightarrow \infty} x_{n}=x$ or $x_{n} \rightarrow x$;

(ii) $\left\{x_{n}\right\}_{n \geq 1}$ is a Cauchy sequence if for every $c \in E$ with $0 \ll c$ there is a natural number $N$, such that $d\left(x_{n}, x_{m}\right) \ll c$ for all $n, m \geq N$;

(iii) $(X, d)$ is a complete cone metric space if every Cauchy sequence in $X$ is convergent in $X$.

Lemma 1.6 (see [1]). Let $(X, d)$ be a CMS, let $P$ be a normal cone with normal constant $K$, and let $\left\{x_{n}\right\}$ be a sequence in $X$. Then,

(i) the sequence $\left\{x_{n}\right\}$ converges to $x$ if and only if $d\left(x_{n}, x\right) \rightarrow 0$ (or equivalently $\left\|d\left(x_{n}, x\right)\right\| \rightarrow$ $0)$;

(ii) the sequence $\left\{x_{n}\right\}$ is Cauchy if and only if $d\left(x_{n}, x_{m}\right) \rightarrow 0$ (or equivalently $\left\|d\left(x_{n}, x_{m}\right)\right\| \rightarrow$ $0)$;

(iii) the sequence $\left\{x_{n}\right\}$ converges to $x$ and the sequence $\left\{y_{n}\right\}$ converges to $y$ then $d\left(x_{n}, y_{n}\right) \rightarrow$ $d(x, y)$.

Lemma 1.7 (see $[1,2])$. Let $(X, d)$ be a CMS over a cone $P$ in E. Thenone has the following.

(1) $\operatorname{Int}(P)+\operatorname{Int}(P) \subseteq \operatorname{Int}(P)$ and $\lambda \operatorname{Int}(P) \subseteq \operatorname{Int}(P), \lambda>0$.

(2) If $c \gg 0$, then there exists $\delta>0$ such that $\|b\|<\delta$ implies $b \ll c$.

(3) For any given $c \gg 0$ and $c_{0} \gg 0$ there exists $n_{0} \in \mathbb{N}$ such that $c_{0} / n_{0} \ll c$.

(4) If $a_{n}, b_{n}$ are sequences in $E$ such that $a_{n} \rightarrow a, b_{n} \rightarrow b$ and $a_{n} \leq b_{n}$ for all $n \geq 1$, then $a \leq b$.

Definition 1.8 (see [12]). $P$ is called minihedral cone if $\sup \{x, y\}$ exists for all $x, y \in E$, and strongly minihedral if every subset of $E$ which is bounded from above has a supremum.

It is easy to see that every strongly minihedral normal cone is regular.

Example 1.9. Let $E=C[0,1]$ with the supremum norm and $P=\{f \in E: f \geq 0\}$. Then $P$ is a cone with normal constant $M=1$ which is not regular. This is clear, since the sequence $x^{n}$ is monotonicly decreasing, but not uniformly convergent to 0 . Thus, $P$ is not strongly minihedral. It is easy to see that the cone mentioned in Example 1.4 is strongly minihedral. 
Definition 1.10 (see [1]). Let $(X, d)$ be a CMS and $A \subset X$. $A$ is said to be sequentially compact if for any sequence $\left\{x_{n}\right\}$ in $A$ there is a subsequence $\left\{x_{n_{k}}\right\}$ of $\left\{x_{n}\right\}$ such that $\left\{x_{n_{k}}\right\}$ is convergent in $A$.

Remark 1.11 (see [6]). Every cone metric space $(X, d)$ is a topological space which is denoted by $\left(X, \tau_{c}\right)$. Moreover, a subset $A \subset X$ is sequentially compact if and only if $A$ is compact.

\section{Main Results}

Let $(X, d)$ be a CMS, $C \subset X$, and $\varphi: C \rightarrow E$ a function on $X$. Then, the function $\varphi$ is called a lower semicontinuous (l.s.C) on $C$ whenever

$$
\lim _{n \rightarrow \infty} x_{n}=x \Longrightarrow \varphi(x) \leq \lim _{n \rightarrow \infty} \inf \varphi\left(x_{n}\right):=\operatorname{supinf}_{n \geq 1} \varphi\left(x_{m}\right)
$$

Also, let $T: C \rightarrow C$ be an arbitrary selfmapping on $C$ such that

$$
d(x, T x) \leq \varphi(x)-\varphi(T x) \quad \forall x \in X
$$

Then, $T$ is called a Caristi map on $(X, d)$.

The following Lemma will be used to prove the next results.

Lemma 2.1. If $\left\{c_{n}\right\}$ is a decreasing sequence (via the partial ordering obtained by the closed cone P) such that $c_{n} \rightarrow u$, then $u=\inf \left\{c_{n}: n \in \mathbb{N}\right\}$.

Proof. Since $\left\{c_{n}\right\}$ is an increasing sequence, $c_{m}-c_{n} \in P$, for $n \geq m$ and $c_{m}-c_{n} \rightarrow c_{m}-u$, for all $m$. Then closeness of $P$ implies that $u \leq c_{m}$ for all $m$. To see that $u$ is the greatest lower bound of $\left\{c_{n}\right\}$, assume that some $v \in E$ satisfies $c_{m} \geq v$ for all $m$. From $\left(c_{m}-v\right) \rightarrow(u-v)$ and the closeness of $P$ we get $(u-v) \in P$ or $v \leq u$ which shows that $u=\inf \left\{c_{n}: n \in \mathbb{N}\right\}$.

Proposition 2.2. Let $(X, d)$ be a compact CMS, $P$ a strongly minihedral cone, and $\varphi: X \rightarrow P \subset E$ a lower semicontinuous (l.s.c) function. Then, $\varphi$ attains a minimum on $X$.

Proof. Let $u=\inf \{\varphi(x): x \in X\}$ which exists by strong minihedrality. For each $n \in N$, there is an $x_{n} \in X$ such that $\varphi\left(x_{n}\right)-u \ll c / n$, where $c \in \operatorname{int} P$. Since $X$ is compact, then $\left\{x_{n}\right\}$ has a convergent subsequence. Let $\left\{y_{n}\right\}$ be this sequence and let $y=\lim y_{n}$.

From the definition of lower semicontinuity and Lemma 2.1 it follows that

$$
\varphi(y) \leq \lim _{n \rightarrow \infty} \inf \varphi\left(y_{n}\right) \ll \lim _{n \rightarrow \infty} \inf \left(u+\frac{c}{n}\right)=u
$$

But then, by the definition of $u, \varphi\left(x_{0}\right) \leq \varphi(x)$ for all $x \in X$. This completes the proof.

Theorem 2.3. Let $(X, d)$ be a CMS, $C$ a compact subset of $X, P$ a strongly minihedral normal cone, and $\varphi: C \rightarrow P \subset E$ a lower semicontinuous (l.s.c) function. Then, each selfmap $T: C \rightarrow C$ satisfying (2.2) has a fixed point in X. 
Proof. By Proposition 2.2, $\varphi$ attains its minimum at some point of $C$, say $u \in C$. Since $u$ is the minimum point of $\varphi$, we have $\varphi(T u) \geq \varphi(u)$. By (2.2),

$$
0 \leq d(u, T u) \leq \varphi(u)-\varphi(T u) \leq 0 .
$$

Thus, $d(u, T u)=0$ and so $T u=u$.

The following theorem is an extension of the result of Caristi ([10, Theorem $\left.2.1^{\prime}\right]$ ).

Theorem 2.4. Let $(X, d)$ be a complete CMS, $P$ a strongly minihedral normal cone, and $\varphi: X \rightarrow$ $P \subset E$ a lower semicontinuous (l.s.c) function. Then, each selmap $T: X \rightarrow X$ satisfying (2.2) has a fixed point in $\mathrm{X}$.

Proof. Let $P$ have the normal constant $K$. Let $S(x):=\{z \in X: d(x, z) \leq \varphi(x)-\varphi(z)\}$ and $\alpha(x):=\inf \{\varphi(z): z \in S(x)\}$ for all $x \in X$. Since $x \in S(x), S(x) \neq \emptyset$ and so $0 \leq \alpha(x) \leq \varphi(x)$.

For $x \in X$, set $x_{1}:=x$ and construct a sequence $x_{1}, x_{2}, x_{3}, \ldots, x_{n}, \ldots$ in the following way: let $x_{n+1} \in S\left(x_{n}\right)$ be such that $\varphi\left(x_{n+1}\right) \leq \alpha\left(x_{n}\right)+c_{0} / n$, where $c_{0} \in \operatorname{Int}(P) \neq \emptyset$. Thus, one can observe that

$$
\begin{aligned}
& \text { (i) } d\left(x_{n}, x_{n+1}\right) \leq \varphi\left(x_{n}\right)-\varphi\left(x_{n+1}\right), \\
& \text { (ii) } \alpha\left(x_{n}\right) \leq \varphi\left(x_{n+1}\right) \leq \alpha\left(x_{n}\right)+c_{0} / n
\end{aligned}
$$

for all $n \geq 1$. Note that, (i) implies that the sequence $\left\{\varphi\left(x_{n}\right)\right\}$ is a decreasing sequence in $E$ and $P$ is regular cone. So, the sequence $\left\{\varphi\left(x_{n}\right)\right\}$ is convergent. Thus, for each $\varepsilon>0$, there exists $N_{\varepsilon}$ such that $\left\|\varphi\left(x_{m}\right)-\varphi\left(x_{n}\right)\right\|<\varepsilon / K$ for all $n, m \geq N_{\varepsilon}$. For $m \geq n$, the triangular inequality implies that

$$
d\left(x_{n}, x_{m}\right) \leq \sum_{j=n}^{m-1} d\left(x_{j}, x_{j+1}\right) \leq \varphi\left(x_{n}\right)-\varphi\left(x_{m}\right)
$$

Hence, $\|d(x, y)\| \leq K\left\|\varphi\left(x_{n}\right)-\varphi\left(x_{m}\right)\right\|<K(\varepsilon / K)=\varepsilon$. By Lemma 1.6, $\left\|d\left(x_{n}, x_{m}\right)\right\| \rightarrow 0$ yields that the sequence $\left\{x_{n}\right\}$ is a Cauchy in $X$. Completeness of $(X, d)$ implies that the sequence $\left\{x_{n}\right\}$ is convergent to some point in $X$, say $y$.

By (2.5), $\varphi\left(x_{n}\right)-\varphi\left(x_{m}\right)-d\left(x_{m}, x_{n}\right) \in P$ and so

$$
\varphi\left(x_{m}\right) \leq \varphi\left(x_{n}\right)-d\left(x_{m}, x_{n}\right)
$$

for all $m \geq n$. By regarding (2.6), Lemma 1.6, and lower semicontinuity of the function $\varphi$, one can obtain that

$$
\varphi(y) \leq \lim _{m \rightarrow \infty} \inf \varphi\left(x_{m}\right) \leq \lim _{m \rightarrow \infty} \inf \left[\varphi\left(x_{n}\right)-d\left(x_{m}, x_{n}\right)\right]=\varphi\left(x_{n}\right)-d\left(x_{n}, y\right)
$$

for all $n \geq 1$. Thus,

$$
0 \leq d\left(x_{n}, y\right) \leq \varphi\left(x_{n}\right)-\varphi(y)
$$


for all $n \geq 1$. Hence, $y \in S\left(x_{n}\right)$ and it is trivial that $\varphi\left(x_{n}\right) \leq \varphi(y)$ for all $n \geq 1$. Note that (ii) implies that

$$
\alpha:=\lim _{n \rightarrow \infty} \alpha\left(x_{n}\right)=\lim _{n \rightarrow \infty} \varphi\left(x_{n}\right)
$$

Thus, $\alpha \leq \varphi\left(x_{n}\right)$ for all $n \geq 1$. On the other hand, by lower semicontinuity of $\varphi$ and (2.9), one can obtain that

$$
\varphi(y) \leq \lim _{n \rightarrow \infty} \inf \varphi\left(x_{n}\right)=\alpha
$$

Therefore, $\alpha=\varphi(y)$.

Since $y \in S\left(x_{n}\right)$ for each $n \geq 1$ and $T y \in S(y)$, the following inequalities are obtained:

$$
d\left(x_{n}, T y\right) \leq d\left(x_{n}, y\right)+d(y, T y) \leq \varphi\left(x_{n}\right)-\varphi(y)+\varphi(y)-\varphi(T y)=\varphi\left(x_{n}\right)-\varphi(T y)
$$

Hence, $T y \in S\left(x_{n}\right)$ for all $n \geq 1$. This implies that $\alpha\left(x_{n}\right) \leq \varphi(T y)$ for all $n \geq 1$.

By (2.9), $\varphi(T y) \geq \alpha$ is obtained. As $\varphi(T y) \leq \varphi(y)$ is observed by (2.2) and that $\varphi(y)=\alpha$, then

$$
\varphi(y)=\alpha \leq \varphi(T y) \leq \varphi(y)
$$

is achieved. Hence, $\varphi(T y)=\varphi(y)$. Finally, by (2.2) we have $T y=y$.

The following theorem is a generalization of the result in [11].

Theorem 2.5. Let $\varphi: X \rightarrow E$ be a l.s.c function on a complete CMS, where $P$ is a strongly minihedral normal cone. If $\varphi$ is bounded below, then there exits $y \in X$ such that

$$
\varphi(y)<\varphi(x)+d(y, x) \quad \forall x \in X \text { with } x \neq y
$$

Proof. It is enough to show that the point $y$, obtained in Theorem 2.4, satisfies the statement of the theorem. Following the same notation in the proof of Theorem 2.4, it is needed to show that $x \notin S(y)$ for $x \neq y$. Assume the contrary that for some $z \neq y$, we have $z \notin S(y)$. Then, $0<$ $d(y, z) \leq \varphi(y)-\varphi(z)$ implies $\varphi(z)<\varphi(y)=\alpha$. By triangular inequality,

$$
d\left(x_{n}, z\right) \leq d\left(x_{n}, y\right)+d(y, z) \leq \varphi\left(x_{n}\right)-\varphi(y)+\varphi(y)-\varphi(z)=\varphi\left(x_{n}\right)-\varphi(z),
$$

which implies that $z \in S\left(x_{n}\right)$ and thus $\alpha\left(x_{n}\right) \leq \varphi(y)$ for all $n \geq 1$. Taking the limit when $n$ tends to infinity, one can obtain $\alpha \leq \varphi(z)$, which is in contradiction with $\varphi(z)<\varphi(y)=\alpha$. Thus, for any $x \in X, x \neq y$ implies $x \notin S(y)$, that is,

$$
x \neq y \Longrightarrow d(y, x)>\varphi(y)-\varphi(x) .
$$

Let $d_{x}: X \rightarrow E$ be defined by $d_{x}(y):=d(x, y)$. 
Theorem 2.6. Let $(X, d)$ be a sequentially complete $Q C M S$ and let $P$ be a strongly minihedral normal cone. Assume that for each $x \in X$, the function $d_{x}$ defined above is continuous on $X$ and $\mathcal{F}$ is a family of mappings $f: X \rightarrow X$. If there exists a l.s.c function $\varphi: X \rightarrow P$ such that

$$
d(x, f(x)) \leq \varphi(x)-\varphi(f(x)), \quad \forall x \in X, \forall f \in \mathcal{F}
$$

then for each $x \in X$ there is a common fixed point $u$ of $\mp$ such that

$$
d(x, u) \leq \varphi(x)-s, \quad \text { where } s=\inf \{\varphi(x): x \in X\} .
$$

Proof. Let $P$ be strongly minihedral normal cone with normal constant $K$. First note that strong minihedrality of $P$ guarantees that $s$ exists. Let $S(x):=\{z \in X: d(x, y) \leq \varphi(x)-\varphi(z)\}$ and $\alpha(x):=\{\varphi(z): z \in S(x)\}$ for all $x \in X$. Note that $x \in S(x)$, so $S(x) \neq \emptyset$ and also $0 \leq \alpha(x) \leq \varphi(x)$.

For $x \in X$, set $x_{1}:=x$ and construct a sequence $x_{1}, x_{2}, x_{3}, \ldots, x_{n}, \ldots$ as in the proof of Theorem 2.4: $x_{n+1} \in S\left(x_{n}\right)$ such that $\varphi\left(x_{n+1}\right) \leq \alpha\left(x_{n}\right)+c_{0} / n, c_{0} \gg 0$. Thus, one can observe that for each $n$,

(i) $d\left(x_{n}, x_{n+1}\right) \leq \varphi\left(x_{n}\right)-\varphi\left(x_{n+1}\right)$,

(ii) $\alpha\left(x_{n}\right) \leq \varphi\left(x_{n+1}\right) \leq \alpha\left(x_{n}\right)+c_{0} / n$.

Similar to the proof of Theorem 2.4, (ii) implies that

$$
\alpha:=\lim _{n \rightarrow \infty} \alpha\left(x_{n}\right)=\lim _{n \rightarrow \infty} \varphi\left(x_{n}\right)
$$

Also, by using the same method in the proof of Theorem 2.4, it can be shown that $\left\{x_{n}\right\}$ is a Cauchy sequence and converges to some $y \in X$ and $\varphi(y)=\alpha$.

We shall show that $f(y)=y$ for all $f \in \mathcal{F}$. Assume the contrary that there is $f \in \mathcal{F}$ such that $f(y) \neq y$. Then (2.16) with $x=y$ implies that $\varphi(f(y))<\varphi(y)=\alpha$. Thus, by definition of $\alpha$, there is $n \in \mathbb{N}$ such that $\varphi(f(y))<\alpha\left(x_{n}\right)$. Since $y \in S\left(x_{n}\right)$,

$$
d\left(x_{n}, f(y)\right) \leq d\left(x_{n}, y\right)+d(y, f(y)) \leq\left[\varphi\left(x_{n}\right)-\varphi(y)\right]+[\varphi(y)-\varphi(f(y))]=\varphi\left(x_{n}\right)-\varphi(f(y)),
$$

which implies that $f(y) \in S\left(x_{n}\right)$. Hence $\alpha\left(x_{n}\right) \leq \varphi(f(y))$ which is in a contradiction with $\varphi(f(y))<\alpha\left(x_{n}\right)$. Thus, $f(y)=y$ for all $f \in \mathcal{F}$. Since $y \in S\left(x_{n}\right)$, we have

$$
d\left(x_{n}, y\right) \leq \varphi\left(x_{n}\right)-\varphi(y) \leq \varphi\left(x_{n}\right)-\inf \{\varphi(z): z \in X\}=\varphi(x)-s
$$

is obtained. 
The following theorem is a generalization of [13, Theorem 2.2].

Theorem 2.7. Let $A$ be a set, $(X, d)$ as in Theorem 2.6, $g: A \rightarrow X$ a surjective mapping, and $\mathcal{F}=\{f\}$ a family of arbitrary mappings $f: A \rightarrow X$. If there exists a l.c.s. function $\varphi: X \rightarrow P$ such that

$$
d(g(a), f(a)) \leq \varphi(g(a))-\varphi(f(a)), \quad \forall f \in \mathcal{F}
$$

and each $a \in A$, then $g$ and $\mathcal{F}$ have a common coincidence point, that is, for some $b \in A, g(b)=f(b)$ for all $f \in \mathcal{F}$.

Proof. Let $x$ be arbitrary and $y \in X$ as in Theorem 2.6. Since $g$ is surjective, for each $x \in X$ there is some $a=a(x)$ such that $g(a)=x$. Let $f \in \mathcal{F}$ be a fixed mapping. Define by $f$ a mapping $h=h(f)$ of $X$ into itself such that $h(x)=f(a)$, where $a=a(x)$, that is, $g(a)=x$. Let $\mathscr{H}$ be a family of all mappings $h=h(f)$. Then, (2.21) yields that

$$
d(x, h(x)) \leq \varphi(x)-\varphi(h(x)), \quad \forall h \in \mathscr{\ell} .
$$

Thus, by Theorem 2.6, $y=h(y)$ for all $h \in \mathcal{H}$. Hence $g(b)=f(b)$ for all $f \in \mathcal{F}$, where $b=b(y)$ is such that $g(b)=y$.

\section{Acknowledgment}

This work is partially supported by the Scientific and Technical Research Council of Turkey.

\section{References}

[1] L.-G. Huang and X. Zhang, "Cone metric spaces and fixed point theorems of contractive mappings," Journal of Mathematical Analysis and Applications, vol. 332, no. 2, pp. 1468-1476, 2007.

[2] Sh. Rezapour and R. Hamlbarani, "Some notes on the paper: "cone metric spaces and fixed point theorems of contractive mappings"," Journal of Mathematical Analysis and Applications, vol. 345, no. 2, pp. 719-724, 2008.

[3] D. Turkoglu and M. Abuloha, "Cone metric spaces and fixed point theorems in diametrically contractive mappings," Acta Mathematica Sinica, English Series, submitted.

[4] D. Turkoglu, M. Abuloha, and T. Abdeljawad, "KKM mappings in cone metric spaces and some fixed point theorems," Nonlinear Analysis: Theory, Methods and Applications, vol. 72, no. 1, pp. 348-353, 2010.

[5] İ. Şahin and M. Telci, "Fixed points of contractive mappings on complete cone metric spaces," Hacettepe Journal of Mathematics and Statistics, vol. 345, pp. 719-724, 2008.

[6] Sh. Rezapour, M. Derafshpour, and R. Hamlbarani, "A Review on Topological Properties of Cone Metric Spaces," in Analysis, Topology and Applications 2008 (ATA2008), Technical Faculty, Cacak, University of Kragujevac Vrnjacka Banja, Serbia, the 30th of May to the 4th of June, 2008.

[7] R. H. Haghi and Sh. Rezapour, "Fixed points of multifunctions on regular cone metric spaces," Expositiones Mathematicae. In press.

[8] Sh. Rezapour and R. H. Haghi, "Fixed point of multifunctions on cone metric spaces," Numerical Functional Analysis and Optimization, vol. 30, no. 7-8, pp. 825-832, 2009.

[9] D. Klim and D. Wardowski, "Dynamic processes and fixed points of set-valued nonlinear contractions in cone metric spaces," Nonlinear Analysis: Theory, Methods and Applications, vol. 71, no. 11, pp. 5170$5175,2009$.

[10] J. Caristi, "Fixed point theorems for mappings satisfying inwardness conditions," Transactions of the American Mathematical Society, vol. 215, pp. 241-251, 1976. 
[11] I. Ekeland, "Sur les problèmes variationnels," Comptes Rendus de l'Académie des Sciences, vol. 275, pp. A1057-A1059, 1972.

[12] K. Deimling, Nonlinear Functional Analysis, Springer, Berlin, Germany, 1985.

[13] L. B. Cirić, “On a common fixed point theorem of a Greguš type," Publications de l' Institut Mathématique, vol. 49, no. 63, pp. 174-178, 1991. 